\title{
Unusual Presentation of a Left Testicular Carcinoid
}

\author{
Amruth R. Palla ${ }^{a}$ Thomas Hogan $^{b}$ Sindhu Singh ${ }^{b}$ \\ ${ }^{a}$ Department of Internal Medicine, Danbury Hospital, Danbury, Conn., and ${ }^{\text {b Section of }}$ \\ Hematology and Oncology, Department of Internal Medicine, Mary Babb Randolph \\ Cancer Hospital, West Virginia University, Morgantown, W.Va., USA
}

\section{Key Words}

Neuroendocrine tumors · Testicular carcinoid

\begin{abstract}
Testicular carcinoid generally presents either with a palpable (painful or painless) or rarely a nonpalpable mass (when it is diagnosed incidentally on scrotal imaging) of the involved testis. An ipsilateral testicular carcinoid presenting exclusively with contralateral testicular symptoms has never been described in the literature to date. We report a case of nonpalpable left testicular carcinoid diagnosed incidentally on a sonogram done for right testicular pain and swelling, with conspicuous absence of left testicular symptoms. This case highlights the importance of recognizing such an atypical presentation of testicular carcinoid.
\end{abstract}

\section{Introduction}

Carcinoid tumors are neuroendocrine tumors originating from the enterochromaffin cells primarily of the gut and also occasionally from the pulmonary, renal or gonadal (ovaries and testes) tissues [1]. They secrete various neurohumoral chemicals including serotonin, histamine and kallikerin which produce the clinical manifestations of carcinoid syndrome (red hot flushing of the face, neck and upper chest, severe and debilitating diarrhea, abdominal pain, palpitations, bronchospasm) [1]. Carcinoid tumors of the testes are uncommon with only 1-3\% [2] of them being associated with carcinoid syndrome and hence their preoperative clinical suspicion is extremely low.

\section{Case Presentation}

A 36-year-old man with no significant past medical history returned to the urology clinic with nocturia, dysuria, and persistent right testicular pain despite 1 month of analgesic therapy. An outpatient scrotal sonogram done 1 month before for this reason showed a 3-mm hypoechoic left testicular mass and some punctate calcifications but no masses or other pathology of the right testicle 
(fig. 1), and the patient was discharged on analgesics. Furthermore, he was noted to have swelling and some tenderness over the right testicular area. The left testicular examination was nondescript with no palpable masses. The patient denied any fever, chills, abdominal pain or any other symptoms. A repeat scrotal sonogram was obtained and showed a stable left testicular mass with suspicion for malignancy and right testicular findings similar to those on the previous sonogram. In view of these findings, the patient underwent an elective left-sided orchiectomy, and pathology revealed a carcinoid tumor ( $\underline{\text { fig. } 2)}$ ) with positive chromogranin, synaptophysin (fig. 3) and CD117 reactivity on immunohistochemical analysis. The tumor was unifocal and no other concurrent pathology (such as teratoma) was seen. CT scans of the chest, abdomen, and pelvis were unremarkable. Laboratory tests done 1 month after surgery showed an elevated serotonin level. Follow-up serotonin levels after 2 months had normalized.

\section{Discussion}

Testicular carcinoids are rare neoplasms of the testes occurring commonly in the 40-60-year age group [2], though cases involving younger populations have been reported. Only about $3 \%$ of these tumors are associated with carcinoid syndrome. They can either be primary or metastatic. Generally, larger tumors and those associated with carcinoid syndrome are metastatic in origin [2]. About a quarter of the primary testicular carcinoids are mixed with teratoma which then portends an indolent and benign course [2]. Also for this reason a germ cell origin of these tumors has been postulated [3].

The common presentation is a painless or painful testicular mass though sometimes patients may present with a nonpalpable mass found incidentally on imaging [4]. Sonogram shows a hypoechoic mass with punctate calcifications within the testicle [5], and histology shows the typical nesting pattern of neoplastic cells [6]. Definitive diagnosis is done on biopsy post-orchiectomy. Grossly, the tumors are solid, yellow or light brown to tan, well-circumscribed but unencapsulated masses [6]. Immunohistochemical analysis shows reactivity of the tumor cells to various neuroendocrine markers including chromogranin A, synaptophysin, neuron-specific enolase and CD117 [6].

High inguinal radical orchiectomy is curative in cases of benign primary testicular carcinoids [2]. Chemotherapy and radiation have not shown much benefit. The prognosis is poor in case of metastatic carcinoids irrespective of the site of the primary tumor [7]. Delayed recurrence of up to 17 years has been documented making postoperative follow-up essential and which consists of whole-body scanning (CT scan of the chest, abdomen and pelvis) and measurement of urinary 5-HIAA or serum serotonin and chromogranin A levels [2].

\section{Conclusion}

The atypical presentation of ipsilateral testicular carcinoid with contralateral testicular pain, nocturia and dysuria has not been previously reported. It is important to recognize this uncommon presentation to avoid missing or delaying the diagnosis of this rare but ominous clinical entity. 


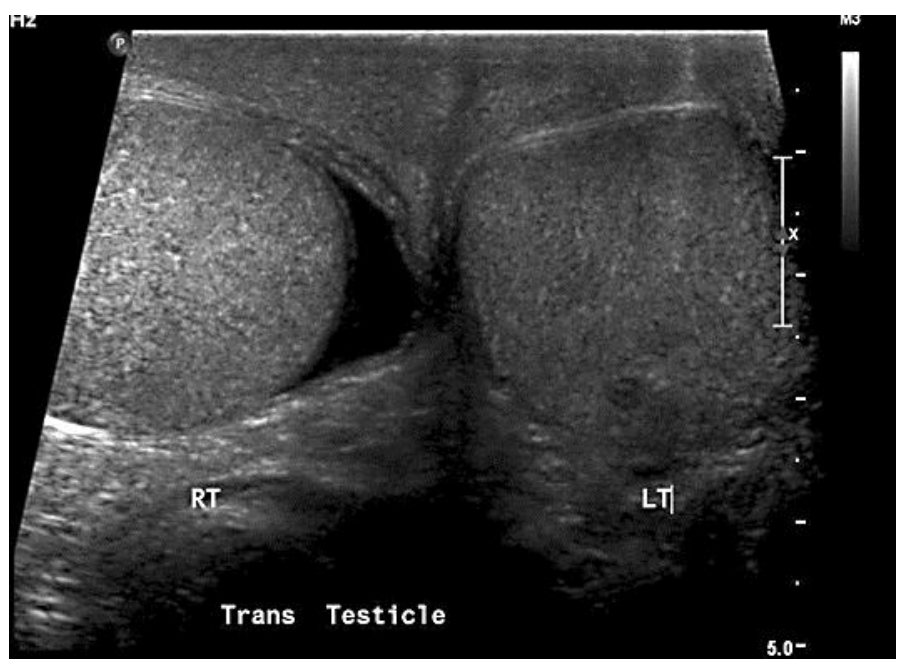

Fig. 1. Sonogram image showing a left testicular tumor and a normal right testis.

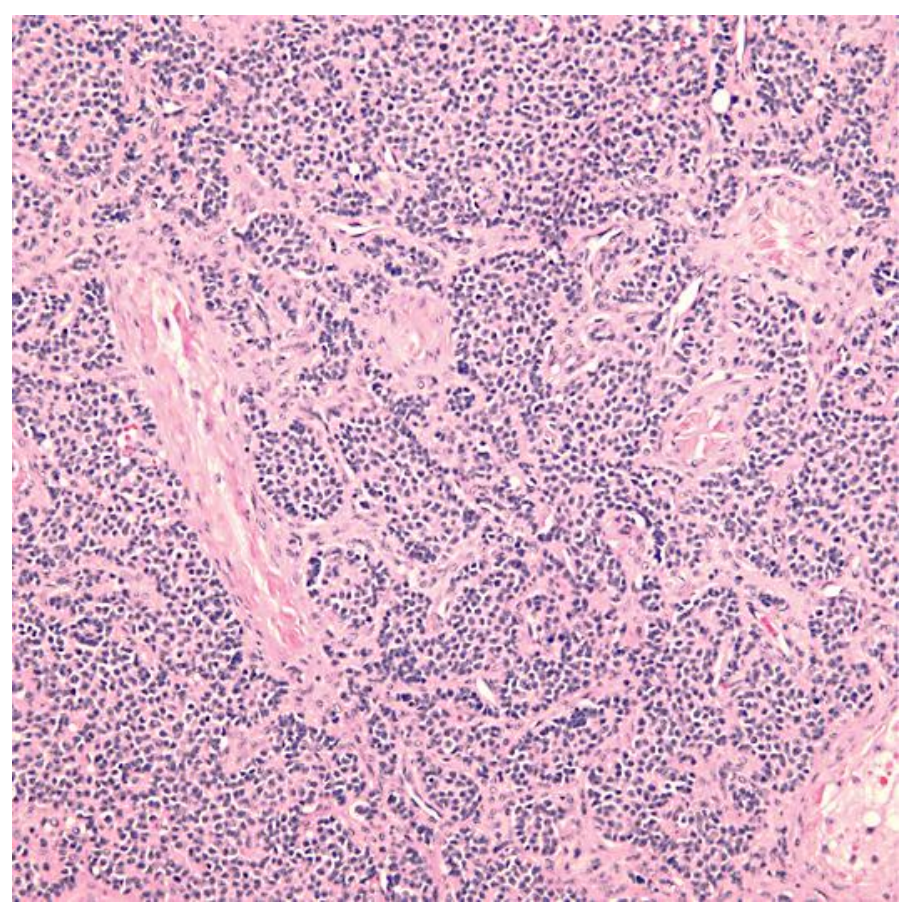

Fig. 2. Biopsy of the left testicle showing evidence of carcinoid tumor with the typical nesting pattern of neoplastic cells. 


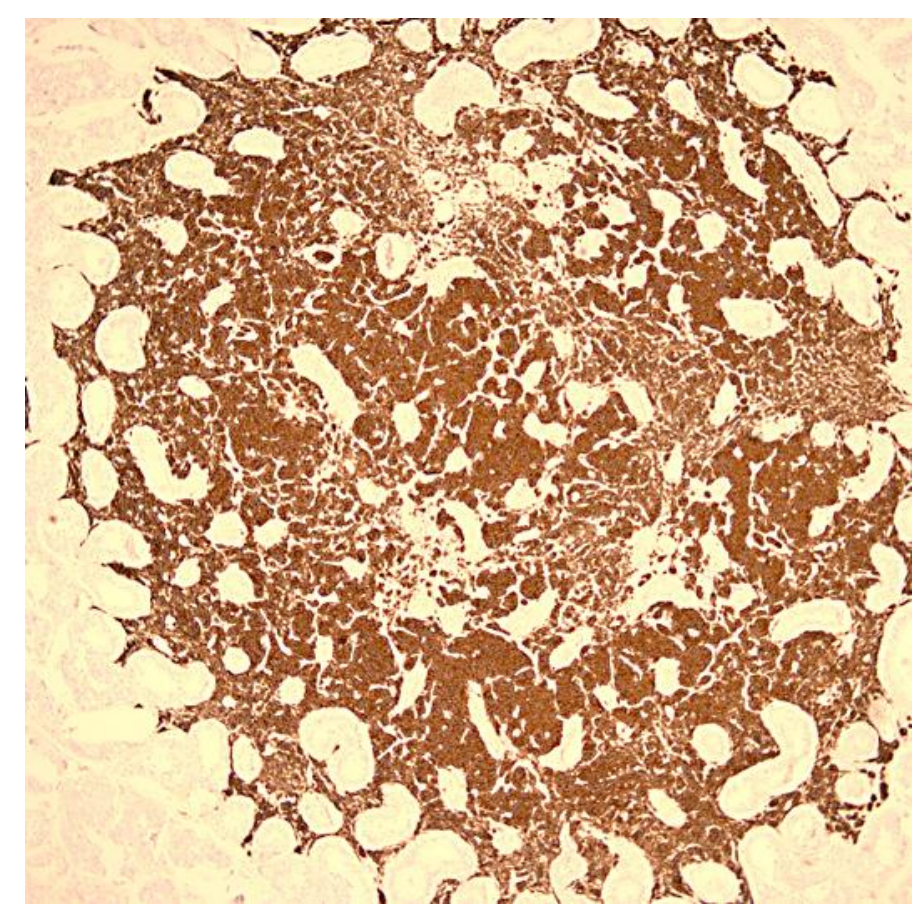

Fig. 3. Immunohistochemical analysis showing reactivity of tumor cells to synaptophysin and chromogranin.

\section{References}

1 Gustafsson BI, Kidd M, Modlin IM: Neuroendocrine tumors of the diffuse neuroendocrine system. Curr Opin Oncol 2008;20:1-12.

2 Thomas JC, Jones JS: Primary carcinoid tumor of the testis found at the time of elective sterilization. J Androl 2004;25:338-339.

-3 Abbosh PH, Zhang S, Maclennan GT, Montironi R, Lopez-Beltran A, Rank JP, Baldridge LA, Cheng L: Germ cell origin of testicular carcinoid tumors. Clin Cancer Res 2008;14:1393-1396.

-4 Frederick LR, Ballek NK, Esplin JA, Kohler TS: Primary carcinoid tumor presenting as a nonpalpable testicular mass. J Clin Oncol 2010;28:e637-e639.

5 Park SB, Kim JK, Cho KS: Imaging findings of a primary bilateral testicular carcinoid tumor associated with carcinoid syndrome. J Ultrasound Med 2006;25:413-416.

6 Reyes A, Moran CA, Suster S, Michal M, Dominguez H: Neuroendocrine carcinomas (carcinoid tumor) of the testis. A clinicopathologic and immunohistochemical study of ten cases. Am J Clin Pathol 2003;120:182-187.

7 Wang WP, Guo C, Berney DM, Ulbright TM, Hansel DE, Shen R, Ali T, Epstein JI: Primary carcinoid tumors of the testis: a clinicopathologic study of 29 cases. Am J Surg Pathol 2010;34:519-524. 